\title{
Strong paramagnon scattering in single atom Pd contacts
}

Schendel, V.; Barreteau, Cyrille; Brandbyge, Mads; Borca, Bogdana; Pentegov, I.; Schlickum, U.; Ternes, M.; Wahl, P.; Kern, K.

Published in:

Physical Review B

Link to article, DOI:

10.1103/PhysRevB.96.035155

Publication date:

2017

Document Version

Publisher's PDF, also known as Version of record

Link back to DTU Orbit

Citation (APA):

Schendel, V., Barreteau, C., Brandbyge, M., Borca, B., Pentegov, I., Schlickum, U., Ternes, M., Wahl, P., \& Kern, K. (2017). Strong paramagnon scattering in single atom Pd contacts. Physical Review B, 96(3), [035155]. https://doi.org/10.1103/PhysRevB.96.035155

\section{General rights}

Copyright and moral rights for the publications made accessible in the public portal are retained by the authors and/or other copyright owners and it is a condition of accessing publications that users recognise and abide by the legal requirements associated with these rights.

- Users may download and print one copy of any publication from the public portal for the purpose of private study or research.

- You may not further distribute the material or use it for any profit-making activity or commercial gain

- You may freely distribute the URL identifying the publication in the public portal 


\title{
Strong paramagnon scattering in single atom Pd contacts
}

\author{
V. Schendel, ${ }^{1,}{ }^{*}$ C. Barreteau, ${ }^{2,3}$ M. Brandbyge, ${ }^{3}$ B. Borca, ${ }^{1}$ I. Pentegov, ${ }^{1}$ U. Schlickum, ${ }^{1}$ M. Ternes, ${ }^{1}$ P. Wahl,,${ }^{4,1}$ and K. Kern ${ }^{1,5}$ \\ ${ }^{1}$ Max-Planck-Institut für Festkörperforschung, Heisenbergstraße 1, 70569 Stuttgart, Germany \\ ${ }^{2}$ Service de Physique de L'Etat Condensé (SPEC), CEA, CNRS, Université Paris-Saclay, \\ CEA Saclay, 91191 Gif-sur-Yvette Cedex, France \\ ${ }^{3}$ Department of Micro- and Nanotechnology, Technical University of Denmark, Ørsteds Plads, \\ Building 345B, DK-2800 Kongens Lyngby, Denmark \\ ${ }^{4}$ SUPA, School of Physics and Astronomy, University of St. Andrews, Scotland, United Kingdom \\ ${ }^{5}$ Institut de Physique, Ecole Polytechnique Fédérale de Lausanne, 1015 Lausanne, Switzerland
}

(Received 8 February 2017; published 31 July 2017)

\begin{abstract}
Among all transition metals, palladium (Pd) has the highest density of states at the Fermi energy at low temperatures yet does not fulfill the Stoner criterion for ferromagnetism. However, close proximity to magnetism renders it a nearly ferromagnetic metal, which hosts paramagnons, strongly damped spin fluctuations. Here we compare the total and the differential conductance of monoatomic contacts consisting of single Pd and cobalt (Co) atoms between Pd electrodes. Transport measurements reveal a conductance for Co of $1 G_{0}$, while for Pd we obtain $2 G_{0}$. The differential conductance of monoatomic Pd contacts shows a reduction with increasing bias, which gives rise to a peculiar $\Lambda$-shaped spectrum. Supported by theoretical calculations, we correlate this finding with the lifetime of hot quasiparticles in $\mathrm{Pd}$, which is strongly influenced by paramagnon scattering. In contrast to this, Co adatoms locally induce magnetic order, and transport through single cobalt atoms remains unaffected by paramagnon scattering, consistent with theory.
\end{abstract}

DOI: 10.1103/PhysRevB.96.035155

Spin fluctuations are believed to provide the pairing glue in unconventional superconductors [1-4]. Therefore, the interaction of magnetic fluctuations with electronic degrees of freedom is critical for a full understanding of unconventional superconductivity [4,5]. A material which in its elemental form exhibits strong magnetic fluctuations yet does not even become a conventional superconductor is palladium [6]. This raises important questions as to how spin fluctuations interact with the conduction band electrons.

Spin fluctuations also play an important role in some of the macroscopic properties of elemental metals: Both palladium $(\mathrm{Pd})$ and platinum $(\mathrm{Pt})$ are not ferromagnetic despite an only partially filled $d$ shell, but belong to a class of materials coined nearly ferromagnetic metals [7]. At low temperatures, Pd possesses the highest density of states (DOS) at the Fermi energy $\left(E_{\mathrm{F}}\right)$ among all transition metals and the Stoner criterion is almost fulfilled [8], bringing it right to the edge to ferromagnetism. In these nearly ferromagnetic metals, strongly damped spin fluctuations, known as paramagnons, have a great impact on macroscopic quantities such as the heat capacity and magnetic susceptibility [9]. Paramagnons in palladium have been commonly observed by means of scattering techniques such as neutron scattering [10] and angle-resolved photoemission [11] within an energy range of about 50-150 meV. Detection at the atomic level, however, has remained elusive.

Paramagnons can be described as magnetic fluctuations of a paramagnetic phase [12]. In contrast to magnons, which are the fluctuations of a magnetically ordered system, paramagnons are collective overdamped modes with only short correlation lengths that appear close to a magnetic instability $[7,13]$. Experimental [8,14-17] and theoretical [18-23] studies have

\footnotetext{
*Corresponding author: verena.schendel@kit.edu
}

explored the possibility of Pd becoming ferromagnetic in nanostructures. Furthermore, very recently the dectection of paramagnetic spin-excitations in single adatoms has been discussed [24]. In this work we study transport through single $\mathrm{Pd}$ and Co adatoms on a Pd(111) surface by scanning tunneling microscopy (STM) and spectroscopy (STS). Differential conductance $(d I / d V)$ spectra were taken at different tip-sample distances $z$ from the tunneling to the contact regime. In Pd contacts, we find that the spectral features show a significant decrease of the conductance with increasing bias, independent of the polarity. By comparison with theoretical calculations this feature can be correlated with the extremely short lifetime of hot quasiparticles in Pd-an effect that we attribute to paramagnon excitations. For Co contacts, the differential conductance is comparatively featureless.

Experiments have been performed on a $\operatorname{Pd}(111)$ single crystal with a home-built UHV-STM operating at $6 \mathrm{~K}$. The sample was cleaned in vacuum with a base pressure of $3 \times 10^{-10}$ mbar by numerous cycles of $\mathrm{Ar}^{+}$sputtering and subsequent annealing. The most frequent bulk contaminants in Pd are sulphur (S) and carbon (C). Upon annealing to temperatures of $1000 \mathrm{~K}$ for extended periods, $\mathrm{S}$ and $\mathrm{C}$ impurities migrate to the surface. $\mathrm{S}$ is removed by sputtering, while $\mathrm{C}$ is removed by exposing the crystal to an oxygen atmosphere $\left(p_{\mathrm{O} 2}=3.0 \times 10^{-7} \mathrm{mbar}\right)$ for $20 \mathrm{~min}$ while heating the sample to temperatures in the range of $650-850 \mathrm{~K}$. The final preparation cycle was carried out in the absence of oxygen and the sample was annealed to $900 \mathrm{~K}$. The apex of the STM tip was covered with Pd by gentle indentation into the surface. Single Pd atoms were released from the tip by moving the tip towards the bare surface until contact was made [Fig. 1(a)]. Co atoms were evaporated from a wire with $99.99 \%$ purity in situ onto the sample being held at $6 \mathrm{~K}$ [Fig. 1(b)]. The two species can be easily distinguished from each other by their apparent heights [Figs. 1(c), 1(d), and 1(e)] 
(a)
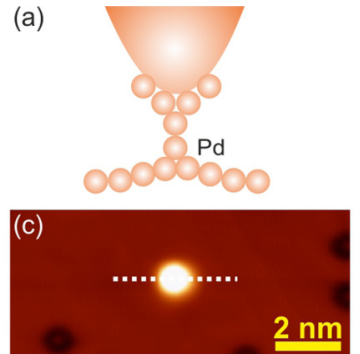

(e)

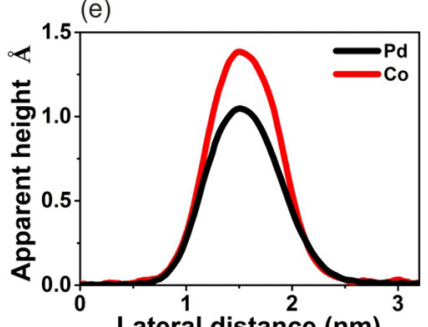

(g)

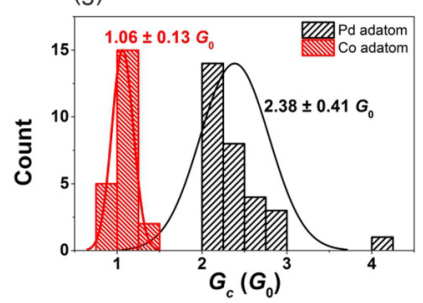

(b)

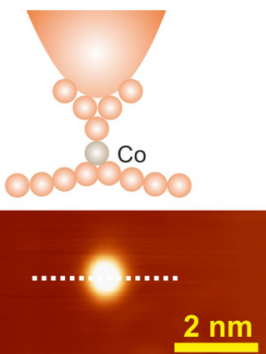

(f)

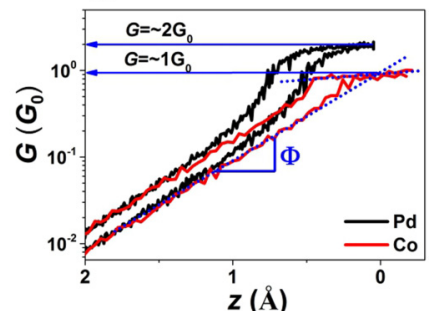

(h)

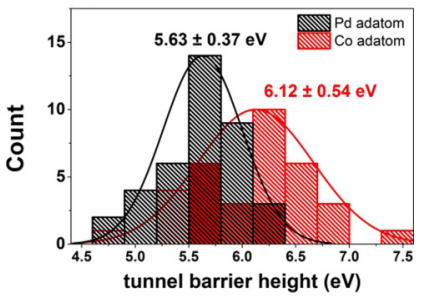

FIG. 1. (a), (b) Sketch of monoatomic Pd (a) and Co (b) contacts. (c), (d) Constant current images of a Pd (c) and a Co (d) adatom acquired on $\operatorname{Pd}(111)$ at $6 \mathrm{~K}(V=0.1 \mathrm{~V}, I=100 \mathrm{pA})$. (e) Apparent height profiles of single Pd and Co atoms. (f) Conductancedisplacement $[G(z)]$ curves taken on Pd (solid black line) and Co (solid red line) show a characteristic conductance of about $2 G_{0}$ for Pd contacts while Co contacts exhibit ones of about $1 G_{0}(V=0.1 \mathrm{~V})$. (g), (h) Conductance and tunnel barrier height measurements on Pd and Co adatoms deposited onto $\operatorname{Pd}(111)$. To extract the mean values, the histograms were fitted with Gaussian functions.

Distance dependent conductance, $G(z)$, measurements were carried out for contacts between the tip and individual $\mathrm{Pd}$ and Co adatoms. From the $G(z)$ curves two regimes are discernible; a tunneling and a contact regime [Fig. 1(f)]. In the tunneling regime, a decrease in $z$ is associated with an exponential increase of the conductance, i.e., $G(z)=$ $G_{0} \exp (-2 \kappa z)$ (with $G_{0}=2 e^{2} / h=77.5 \mu \mathrm{S}$ as the quantum of conductance). The slope $\kappa=\sqrt{\frac{m_{0}}{\hbar^{2}} \Phi}$ is directly related to the local tunnel barrier height $\Phi[25]$. Reducing $z$ further leads to a relaxation of the tip and the surface atoms due to adhesive forces. When contact between the atom at the tip apex and the surface is established, a discontinuous jump in $G(z)$ occurs. This jump arises when the bonding strength between surface and tip apex atom overcomes that between the atoms within the tip. As displayed in Fig. 1(f) for the approach curve on a Co atom, the conductance of the contact $G_{c}$ can be obtained by extrapolating the tunneling regime to the intersection with the contact regime, which is defined as $z=0$. Positive $z$ values denote tunneling, whereas negative ones denote the contact regime.
The measured values of $G_{c}$ and $\Phi$ for both types of adatoms are summarized in Figs. 1(g) and 1(h). For the tip-Pd adatom and tip-Co adatom contacts we found $G_{c}=2.38 \pm 0.41 G_{0}$ and $1.06 \pm 0.13 G_{0}$, respectively. The results for Co adatoms are consistent with previous reports for Co adatoms on noble metal surfaces [26,27]. For Pd contacts, the reported values exhibit variations depending on preparation conditions. For break junctions prepared in vacuum at room temperature, a conductance of $0.5 G_{0}$ was found and interpreted in terms of a single spin-polarized conductance channel [16]. Other measurements carried out on $\mathrm{Pd}$ break junctions at low temperatures and in vacuum reported conductances in good agreement with our values $[28,29]$.

Differential conductance $(d I / d V)$ spectra were obtained on top of $\mathrm{Pd}$ and $\mathrm{Co}$ adatoms starting from the tunneling regime to contact [Figs. 2(a) and 2(b)]. Spectra taken on the Pd adatoms in the tunneling regime give access to the distribution of occupied and unoccupied states near $E_{\mathrm{F}}$, reflecting the local density of states of Pd [9]. However, when the contact regime is reached a drastic change of the feature to a distinct $\Lambda$-shaped peak occurs. Further increasing the conductance of the contact leads to a significant broadening and decrease of the signal strength of the $\Lambda$-like feature. A slight shift of the curves is observed upon decreasing the tip-adatom distance beyond the contact point, which, however, is not systematic and was found to be tip dependent. In this regime, the contact is squeezed which likely causes a rearrangement of the tip atoms which may lead to more asymmetric $I-V$ characteristics. Contrary to these observations the $d I / d V$ spectra taken on Co adatoms show a rather flat signal in the tunneling as well as in the contact regime. The small feature on the cobalt atoms around zero bias might be due to emergent Kondo correlations, which are commonly observed for magnetic adatoms on noble metal surfaces [30,31].

To understand the $\Lambda$ anomaly, we have performed density functional theory (DFT) and electronic transmission calculations in the nonequilibrium Green function (NEGF) formalism with the ATOMISTIX TOOLKIT (ATK) code from QuantumWise [32] and the TRANSIESTA code [33] to model the transport through single cobalt and palladium adatoms. All our
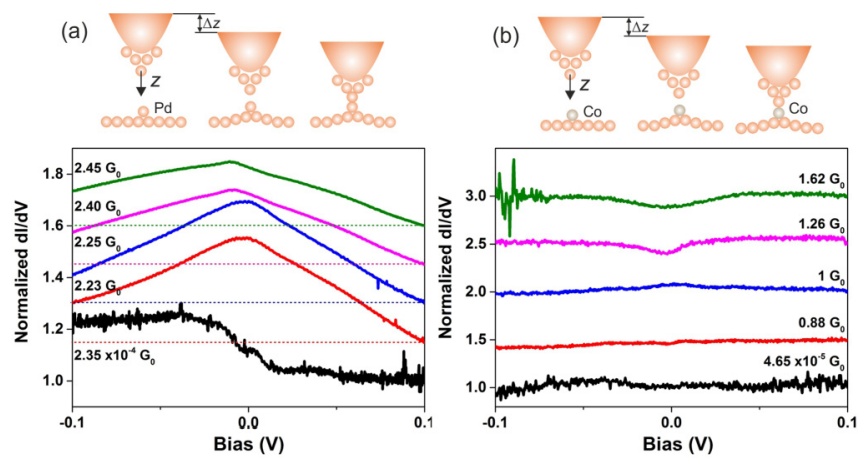

FIG. 2. Differential conductance $(d I / d V)$ acquired on a $\mathrm{Pd}$ adatom (a) and Co adatom (b) deposited on $\operatorname{Pd}(111)$. Spectra were recorded with a lock-in modulation of $2 \mathrm{mV}$ and taken through different heights from tunneling to contact as indicated by the setpoint conductance. Curves are normalized at $V=0.1 \mathrm{~V}$ (horizontal dashed lines) and stacked for clarity. 
calculations were performed within the local density approximation (LDA) using the Perdew-Zunger parametrization [34] since the generalized gradient approximation incorrectly predicts a magnetic ground state for Pd [35]. ${ }^{1}$ We used a single zeta polarized (SZP) basis set and separable norm-conserving Troullier-Martins pseudopotentials [36] with partial core corrections.

We performed DFT calculations for these two systems to establish the differences in the electronic states near $E_{\mathrm{F}}$. We found no evidence of spin polarization for the Pd adatom, while a strong spin polarization develops on the Co adatom with a spin moment of $2.76 \mu_{\mathrm{B}}$. In addition, we find non-negligible spin polarization on neighboring $\mathrm{Pd}$ atoms, indicating that the Co atom locally induces magnetic order [Fig. 3(a)].

Figure 3(b) depicts the projected density of states (PDOS) of the $d$ orbitals for bulk and surface Pd atoms as well as for the adatoms ( $\mathrm{Pd}$ and $\mathrm{Co})$. The $d$ band of $\mathrm{Pd}$ is almost filled and $E_{\mathrm{F}}$ falls into the tail of the $d$ states. Note that when considering the effect of a tip in contact with a Pd adatom we found a smooth decrease of the PDOS around $E_{\mathrm{F}}$, which disfavors the appearance of magnetism [cf. Fig. 3(c)]. For cobalt, the $d$ states exhibit a strong exchange splitting. The states at $E_{\mathrm{F}}$ are dominated by $d$ states of minority spin character.

A series of electronic transport calculations was carried out where the system is divided into three regions: left and right leads and a central region containing the atomic contact. The leads are built from a semi-infinite repetition of three atomic layers with an fcc stacking. A $4 \times 4$ unit cell is used and periodic boundary conditions are applied in the (111) plane. We checked that using a $5 \times 5$ unit cell did not change our results significantly. The central part is made of three layers in contact via a four-atom pyramid whose apex is at a distance $d$ from the adatom. Only the adatom and the pyramid were allowed to relax. A sketch of the system is presented in the inset of Fig. 4(a).

The electronic transmission through a Pd adatom as a function of energy [Fig. 4(a)] depends crucially on the tip-adatom distance $d$, but for distances between 2.5 and $2.9 \AA$ the typical conductances are on the order of (2-2.5) $G_{0}$, which is in the range of the experimental values. It is also of the same order of magnitude as found by Gava et al. [23] for the transmission between two $\operatorname{Pd}(001)$ surfaces connected by a small atomic chain.

The transmission through a magnetic Co adatom drops drastically (by a factor of 2) compared to the case of the $\mathrm{Pd}$ adatom. For the sake of comparison we calculated the transmission through a hypothetical nonmagnetic Co adatom for which the conductance at $E_{\mathrm{F}}$ is close to the one of the Pd adatom, confirming the influence of the local magnetization on the electronic transmission. These theoretical results are perfectly consistent with the experimental ones.

The complete modeling of the differential conductance curves is rather cumbersome, since this would involve the calculation of the electrical current at various bias voltages and then calculating the derivative of $I(V)$. However, in the present

${ }^{1}$ Our LDA calculations predict an equilibrium lattice parameter of $3.87 \AA$ for face centered (fcc) Pd and an onset of magnetism for lattice parameters above $3.96 \AA$ while GGA calculations predict a magnetic bulk at equilibrium. (a)

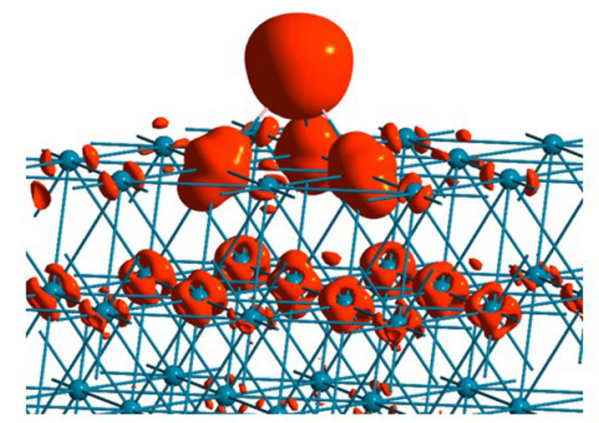

(b)

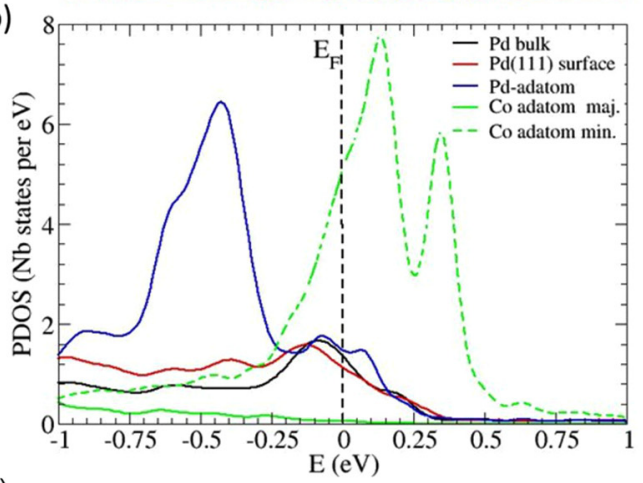

(c)

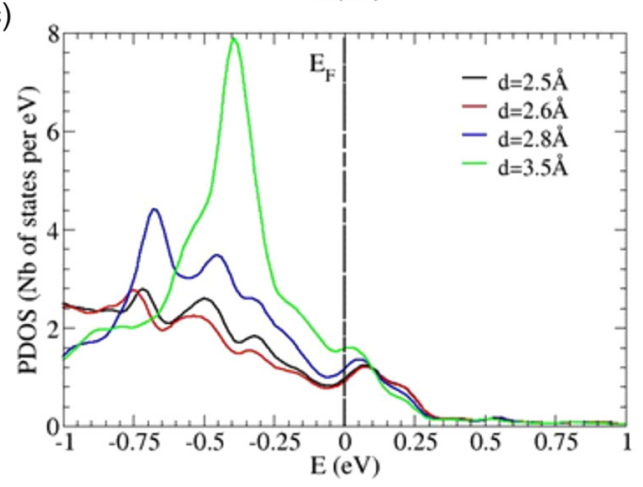

FIG. 3. (a) Real-space distribution of the spin-density isosurface plot of a Co adatom on a $\operatorname{Pd}(111)$ surface. The magnetic moment on the cobalt atom is $2.76 \mu_{\mathrm{B}}$, while the one induced on the palladium atoms is $0.26 \mu_{\mathrm{B}}$ on the nearest neighbors and $0.13 \mu_{\mathrm{B}}$ in the sublayer. (b) Calculated projected density of states (PDOS) on the $d$ orbitals of bulk (black) and (111) surface (red) atoms and Pd (blue) and Co (green) adatoms. In the case of the cobalt adatom the majority spin (full green) and the minority spin (dashed green) are split by a large exchange and the principal PDOS contribution from the majority spin is well below $E_{\mathrm{F}}-1 \mathrm{eV}$, while for palladium there is no magnetism and the two spins are degenerate. (c) Evolution of the DOS with decreasing tip-Pd adatom distance.

case, in the contact regime, for a highly symmetric system (identical leads) and in a very narrow voltage range around the Fermi level, one expects an almost odd $I(V)$ curve and therefore an even $d I / d V$ curve which can safely be approximated by the average transmission, $\frac{1}{2}\left[T\left(E=-\frac{V}{2}\right)+T\left(E=\frac{V}{2}\right)\right]$. Using this approximation we find flat and featureless $d I / d V$ spectra for bias voltages between -0.1 and $0.1 \mathrm{~V}$ at tip-adatom distances between 2.5 and $3 \AA$ for both Co and Pd contacts, which do not reproduce the experimental findings.

The discrepancy between the experimentally observed and calculated conductance spectra can be lifted when taking the finite lifetime of the hot quasiparticles into account. The 

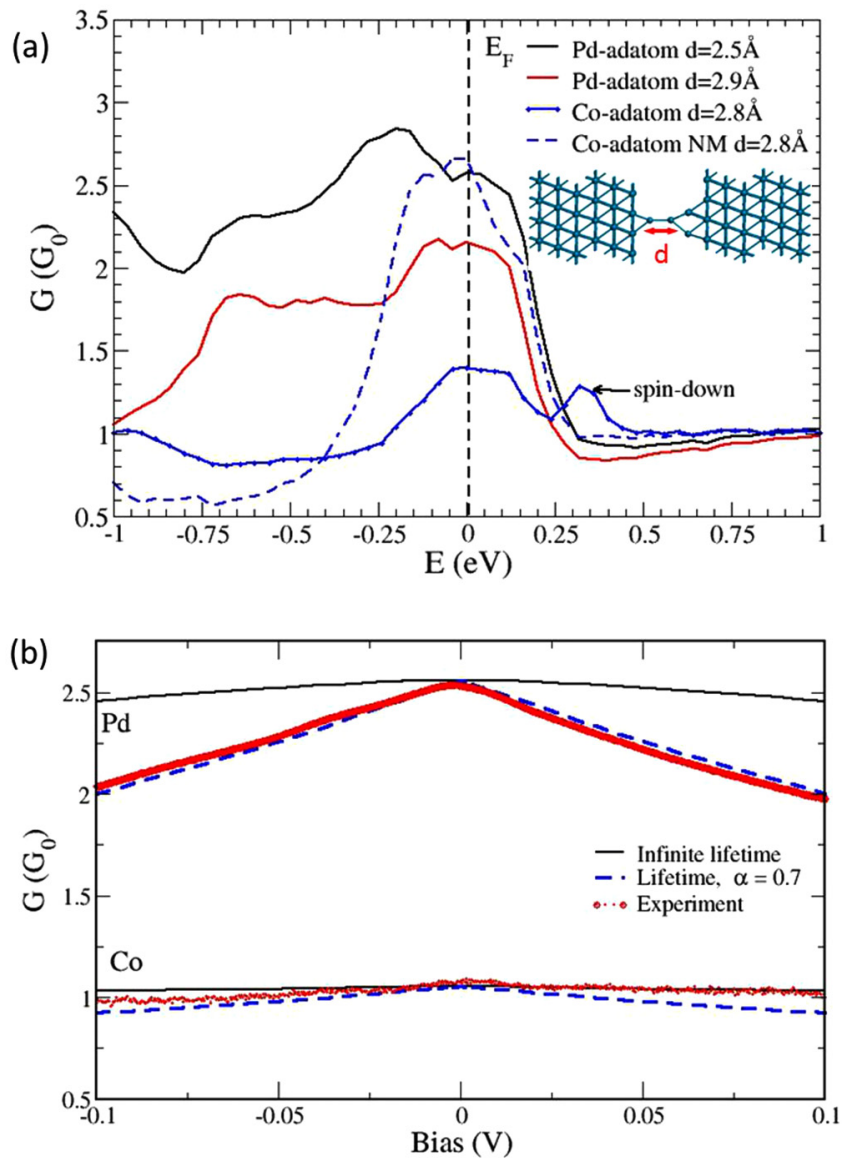

FIG. 4. (a) Calculated electronic transmission through a monatomic $\mathrm{Pd}(\mathrm{Co})$ contact. $d$ is the distance between the adatom and the apex of the tip. In the case of a Co adatom the system is spin polarized. Additionally we have also considered the case of a non-magnetic (NM) Co adatom. The sharp feature above $0.25 \mathrm{eV}$ (indicated by an arrow) originates from the spin-down contribution. The Fermi level is set as the zero of energy. (b) Differential conductance $(d I / d V)$ calculated with and without including a lifetime broadening, $\Gamma(V)=\alpha|V|$, in the electrodes. The parameter $\alpha$ is fitted to the experimental results.

quasiparticle lifetime in Pd has been calculated using various GW-based many-body perturbation theories [37,38] showing considerably shorter lifetimes close to $E_{\mathrm{F}}$ compared to, e.g., $\mathrm{Au}$ or $\mathrm{Cu}$. It also displays a different behavior compared to the free-electron behavior in Fermi liquid theory, $\hbar / \tau=\Gamma \propto$ $\left(E-E_{\mathrm{F}}\right)^{2}$. This is attributed to the role of the $d$ electrons and their finite bandwidth. Early calculations [39] using a finite bandwidth model for an almost full band suggested an electronic level broadening due to spin-fluctuations in $\mathrm{Pd}$ of $\Gamma(E) \approx 0.05\left|E-E_{\mathrm{F}}\right|$. Lifetimes $\tau$ calculated within the $\mathrm{GW}$ approximation [38] result in $\tau \sim 10 \mathrm{fs}$, corresponding to $\Gamma \approx 50 \mathrm{meV}$ for $E-E_{\mathrm{F}} \approx 250 \mathrm{meV}$. Based on this we include an additional bias-dependent contribution to the imaginary part of the self-energy, $\Gamma(V)=\alpha|e V|$, to the $d$ orbitals in the two outermost surface layers in the calculation. This corresponds to the lifetime of electrons injected from the negative electrode that enter $e V$ above the Fermi level in the positive electrode.
The addition of a linear term to the self-energy for the lifetime of quasiparticles accounts for the continuous $\Lambda$ shaped nature of the feature, which indicates that its origin is a collective phenomenon rather than a localized phonon or spin excitation in the atomic contact, where an abrupt jump would be expected.

If we fit the unknown $\alpha$ to the experiments, as shown in Fig. 2(d), we obtain $\alpha \sim 0.7$ corresponding to a lifetime of roughly $10 \mathrm{fs}$ at a bias of $100 \mathrm{mV}$, slightly below the $\mathrm{GW}$ results.

It is interesting to note that similar spectroscopic features have been observed in mesoscopic contacts with conductances on the order of about $100 G_{0}$ and $800 G_{0}$, respectively $[17,28]$. While the details might differ, we expect that the mechanism leading to the suppression of conductance with increasing bias voltage in those contacts is the same.

At large currents the rate at which hot electrons are transmitted through the junction becomes comparable to the decay rate of the quasiparticles, $\tau$, which means that nonequilibrium effects will start to play a role. As a rough estimate these effects will become relevant when $e / I=e /(G V)<\tau=\hbar /(\alpha e V)$, which yields $G / G_{0}>\alpha \pi$. Therefore, when the tip-adatom distance decreases beyond the point of contact and the contact is squeezed, the conductance $G$ increases such that the above expression is fulfilled. With $\alpha=0.7$ this occurs at $G / G_{0} \approx 2.2$. This can rationalize the decrease in the slope of the $\Lambda$-shaped signature observed at larger conductances. Therefore, to evaluate the energy dependence of the lifetime of the quasiparticles, the curve obtained right after the jump to contact was fitted.

Based on the theoretical calculations we attribute the decrease of the conductance with increasing bias to a suppression of the transmission of electrons. With increasing energy, more paramagnons are excited and more charge carriers are reflected. A characteristic feature of paramagnons is that they are overdamped modes, meaning they have short lifetimes and couple strongly to electrons to release their energy. As a consequence, their response in momentum space is broad and not distinctly defined, giving rise to a broad and smeared out feature similar to what we observe. The strongest argument for the presence of paramagnons in palladium is the extremely short lifetime of quasiparticles that is obtained from the fit to the observed spectra. The different slopes of the $d I / d V$ spectra for Pd and Co contacts depicted in Fig. 4(b) are directly related to the magnetism of the Co adatom. While for palladium the $d$ states at the Fermi level contribute substantially to the conductance, for cobalt the exchange coupling pushes the majority- $d$ states below the Fermi level, and thus it is only spin-majority states of $s$ character and spin-minority states of $d$ character which contribute to the transport. The electronic states of $s$ character are only weakly affected by paramagnetic excitations, and therefore the $\Lambda$ shape is suppressed.

In conclusion, we have studied atomic contacts consisting of single $\mathrm{Pd}$ and $\mathrm{Co}$ atoms. Contacts of single $\mathrm{Pd}$ atoms exhibit pronounced $\Lambda$-shaped spectra, which we attribute to strong electron scattering near the contact which limits the lifetime of the charge carriers. This effect is explained by the presence of paramagnons. Conversely, contacts consisting of Co adatoms, which locally induce magnetic order, do not exhibit a similar suppression of conductance. Hence, we have 
demonstrated that signatures of paramagnons, which were commonly investigated by means of scattering techniques that integrate over larger areas, can be detected with local probes. This might expand the experimental tools for the investigation of high-temperature superconductors.
V.S., U.S., and B.B. acknowledge funding by the SFB 767 and the Emmy-Noether-Program of the Deutsche Forschungsgemeinschaft. C.B. and M.B. would like to thank Derek Stewart for providing the pseudopotential of palladium.
[1] D. Fay and J. Appel, Phys. Rev. B 22, 3173 (1980).

[2] T. Moriya, Y. Takahashi, and K. Ueda, J. Phys. Soc. Jpn. 59, 2905 (1990).

[3] N. D. Mathur, F. M. Grosche, S. R. Julian, I. R. Walker, D. M. Freye, R. K. W. Haselwimmer, and G. G. Lonzarich, Nature (London) 394, 39 (1998).

[4] D. J. Scalapino, Rev. Mod. Phys. 84, 1383 (2012).

[5] S. Chi, R. Aluru, S. Grothe, A. Kreisel, U. Singh, B. Andersen, W. Hardy, R. Liang, D. Bonn, S. Burke, and P. Wahl, Nat. Commun. 8, 15996 (2017).

[6] B. Stritzker, Phys. Rev. Lett. 42, 1769 (1979).

[7] G. G. Lonzarich and L. Taillefer, J. Phys. C 18, 4339 (1985).

[8] D. A. van Leeuwen, J. M. van Ruitenbeek, G. Schmid, and L. de Jongh, Phys. Lett. A 170, 325 (1992).

[9] F. M. Mueller, A. J. Freeman, J. O. Dimmock, and A. M. Furdyna, Phys. Rev. B 1, 4617 (1970).

[10] R. Doubble, S. M. Hayden, P. Dai, H. A. Mook, J. R. Thompson, and C. D. Frost, Phys. Rev. Lett. 105, 027207 (2010).

[11] H. Hayashi, K. Shimada, J. Jiang, H. Iwasawa, Y. Aiura, T. Oguchi, H. Namatame, and M. Taniguchi, Phys. Rev. B 87, 035140 (2013).

[12] O. P. Sushkov, Nat. Phys. 10, 339 (2014).

[13] T. Moriya and Y. Takahashi, J. Phys. Colloq. 39, C6 (1978).

[14] X. Teng, W. Q. Han, W. Ku, and M. Hücker, Angew. Chem. Int. Ed. 47, 2055 (2008).

[15] C. Xiao, H. Ding, C. Shen, T. Yang, C. Hui, and H. J. Gao, J. Phys. Chem. C 113, 13466 (2009).

[16] V. Rodrigues, J. Bettini, P. C. Silva, and D. Ugarte, Phys. Rev. Lett. 91, 096801 (2003).

[17] K. Ienaga, H. Takata, Y. Onishi, Y. Inagaki, H. Tsujii, T. Kimura, and T. Kawae, Appl. Phys. Lett. 106, 021605 (2015).

[18] K. Lee, Phys. Rev. B 58, 2391 (1998).

[19] A. Delin, E. Tosatti, and R. Weht, Phys. Rev. Lett. 92, 057201 (2004).

[20] K. M. Smelova, D. I. Bazhanov, V. S. Stepanyuk, W. Hergert, A. M. Saletsky, and P. Bruno, Phys. Rev. B 77, 033408 (2008).
[21] Y. B. Kudasov and A. S. Korshunov, Phys. Lett A 364, 348 (2007).

[22] Y. Sun, J. D. Burton, and E. Y. Tsymbal, Phys. Rev. B 81, 064413 (2010).

[23] P. Gava, A. Dal Corso, A. Smogunov, and E. Tosatti, Eur. Phys. J. B 75, 57 (2010).

[24] J. Ibañez-Azpiroz, M. dos Santos Dias, B. Schweflinghaus, S. Blügel, and S. Lounis, Phys. Rev. Lett. 119, 017203 (2017).

[25] G. Binnig, H. Rohrer, C. Gerber, and E. Weibel, Appl. Phys. Lett. 40, 178 (1982).

[26] N. Néel, J. Kröger, L. Limot, K. Palotas, W. A. Hofer, and R. Berndt, Phys. Rev. Lett. 98, 016801 (2007).

[27] L. Vitali, R. Ohmann, S. Stepanow, P. Gambardella, K. Tao, R. Huang, V. S. Stepanyuk, P. Bruno, and K. Kern, Phys. Rev. Lett. 101, 216802 (2008).

[28] Sz. Csonka, A. Halbritter, G. Mihály, O. I. Shklyarevskii, S. Speller, and H. van Kempen, Phys. Rev. Lett. 93, 016802 (2004).

[29] T. Matsuda and T. Kizuka, Jpn. J. Appl. Phys. 46, 4370 (2007).

[30] M. Ternes, A. J. Heinrich, and W.-D. Schneider, J. Phys. Condens. Matter 21, 053001 (2009).

[31] M. Schneider, L. Vitali, P. Wahl, N. Knorr, L. Diekhoner, G. Wittich, M. Vogelgesang, and K. Kern, Appl. Phys. A 80, 937 (2005).

[32] Atomistix ToolKit version 2015.1, QuantumWise A/S (http://www.quantumwise.com).

[33] M. Brandbyge, J.-L. Mozos, P. Ordejón, J. Taylor, and K. Stokbro, Phys. Rev. B 65, 165401 (2002).

[34] J. P. Perdew and A. Zunger, Phys. Rev. B 23, 5048 (1981).

[35] S. S. Alexandre, M. Mattesini, J. M. Soler, and F. Yndurain, Phys. Rev. Lett. 96, 079701 (2006); A. Delin, E. Tosatti, and R. Weht, ibid. 96, 079702 (2006).

[36] N. Troullier and J. L. Martins, Phys. Rev. B 43, 1993 (1991).

[37] F. Ladstädter, U. Hohenester, P. Puschnig, and C. AmbroschDraxl, Phys. Rev. B 70, 235125 (2004).

[38] V. P. Zhukov, E. V. Chulkov, and P. M. Echenique, Phys. Rev. B 72, 155109 (2005).

[39] J. P. Longo and B. Mitrović, J. Low Temp. Phys. 74, 141 (1989). 